Research Article

\title{
Simultaneous Measurement of In-Plane and Out-of-Plane Displacements Using Talbot Fringe Projection
}

\author{
Haibin Sun $(\mathbb{D})$ and Tingting Liu \\ College of Physics and Electronic Engineering, Taishan University, Tai'an, Shandong 271000, China \\ Correspondence should be addressed to Haibin Sun; sunhbphy@163.com
}

Received 1 September 2019; Revised 12 January 2020; Accepted 3 February 2020; Published 26 February 2020

Academic Editor: E. Bernabeu

Copyright (C) 2020 Haibin Sun and Tingting Liu. This is an open access article distributed under the Creative Commons Attribution License, which permits unrestricted use, distribution, and reproduction in any medium, provided the original work is properly cited.

\begin{abstract}
The fringe projection technique has been widely used in optical measurements. In this paper, we demonstrate a scheme to measure the 3D displacement of a deformed sample using Talbot fringe projection. In this process, we designed a two-dimensional square Talbot hologram. In this approach, we used the basic principle of triangulation, and a computer-controlled liquid crystal spatial light modulator (LC-SLM) was placed in the optical path. The Talbot array hologram was displayed on the LC-SLM screen and projected onto the surface of a sample. Two patterns were recorded: one before and one after deformation. We simultaneously acquired the in-plane and out-of-plane displacements using the digital image correlation (DIC) method. This scheme is simple and easily implemented. Theoretical and experimental results are presented.
\end{abstract}

\section{Introduction}

As an example of a fringe-based technique, fringe projection has been widely used to develop measurement systems at the macroscopic and microscopic levels [1-6]. This technique can be used to generate 3D full-field noncontact topography images of objects with high resolution, high speed, and fast data processing [7]. In fringe projection techniques, fringes are projected onto the surface of a diffusely reflecting sample. Images of the patterns on the surface before and after deformation are then captured using a CCD camera. Sinusoidal fringes are usually employed in fringe projection techniques to acquire the information modulated by the surface of the sample [8]. Linear-, triangular-, and hexagonal-structured patterns have also been used in fringe projection techniques [9]. A phase map is then obtained from the recorded patterns using fringe analysis methods. This phase map can be used to determine the correspondence between the projector image and the camera image of a single-camera or multicamera projector system [10]. To extract the phase information from these fringes, algorithms based on phase shifting, Fourier transform, windowed Fourier transform, and wavelet transform have been developed [11-15].
In optical metrology, the Talbot effect has been exploited for the measurement of displacement and distance [16-20], the measurement of contouring [21-24], and surface roughness $[25,26]$. Linear gratings and circular gratings are commonly employed in applications of the Talbot effect due to their simple patterns and the ease of analysis of the moiré fringes [27]. Based on the encoding of the target surface by a Talbot self-image of a linear grating, Rodriguez-Vera et al. used Talbot-projected fringes to determine the 3D contouring of a diffuse target [28]. They measured the uniform pressure loaded on a clamped latex using Talbot projection profilometry [29].

This report presents the details of a Talbot projection scheme to measure out-of-plane and in-plane displacements simultaneously. We designed a square Talbot array hologram as a projection grating. The Talbot array hologram is displayed on the screen of a phase-only liquid crystal spatial light modulator (LC-SLM) and projected onto a sample. Images of the patterns on the surface of the sample before and after the deformation are captured by a CCD camera. The displacements can be obtained using digital image correlation (DIC). This technique is simple and easily implemented and can be used as an alternative method to 
measure the in-plane and out-of-plane displacements. Theoretical and experimental results are presented.

\section{Principle of Talbot Fringe Projection Technique}

The Talbot fringe projection system follows the basic principle of triangulation, as shown in Figure 1. The optical axis of the imaging system has an angular relationship with the projection system. A laser illuminates an LC-SLM, and a computer-generated Talbot array hologram is written and displayed on the screen of the LC-SLM. The reconstructed image from the Talbot array on the LC-SLM is projected onto a sample. When the LCSLM is placed at an appropriate distance (an integer multiple of the Talbot distance) in front of the sample, a fringe pattern is formed on the surface of the measured sample. A CCD camera is used to record the deformed projection fringe patterns that are modulated by the displacement of the surface of the sample.

When the LC-SLM is illuminated by the laser, the diffracted field replicates an exact image of the Talbot array in a series of equally spaced planes. These Talbot image planes are located at regular distances away from the grating. This distance $Z_{n}$ is determined as follows:

$$
Z_{n}=2 n d^{2} / \lambda
$$

where $d$ is the spatial period of the array, $\lambda$ is the wavelength of the laser, and $n$ is a positive integer called the self-image number. When $n=1$, the associated distance is called the Talbot distance $Z_{T}$.

Figure 2 is a two-dimensional square Talbot array. The designed square Talbot array has a spatial period $d=120 \mu \mathrm{m}$, and the length of the unit square is $a=20 \mu \mathrm{m}$. There are $100-$ unit squares in total. The wavelength of the designed input beam was $632.8 \mathrm{~nm}$, and the corresponding Talbot distance was $45.51 \mathrm{~mm}$.

Figure 3 illustrates the schematic geometry of the Talbot fringe projection system. When the sample is loaded, the speckle originally located at point $E$ moves to point $F$ due to an in-plane displacement. The out-of-plane height of point $C$ leads to the in-plane displacement $u$. From the geometry of Figure 3, the in-plane displacement $u$ can be written as follows:

$$
u=E F=\Delta x=w \cdot \frac{d}{L-w},
$$

where $L$ is the distance between the CCD camera and the reference plane (before the deformation), $w$ is the out-of-plane displacement, and $d$ is the distance between the CCD and the projector. Given that $L \gg w$, equation (2) can be written as follows:

$$
u=w \cdot \frac{d}{L}
$$

In speckle projection profilometry, the in-plane displacement $u$ measured by the DIC method has unit pixels, so the actual physical distance is given by $\Delta x=M u$, where $M$ is the magnification of the imaging system. Thus, the out-ofplane displacement $w$ can be expressed as follows [30]:

$$
w=k u,
$$

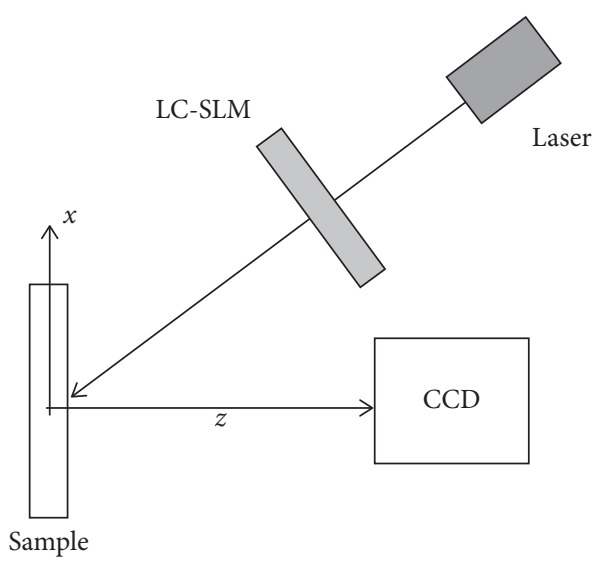

Figure 1: Geometry of the Talbot fringe projection.

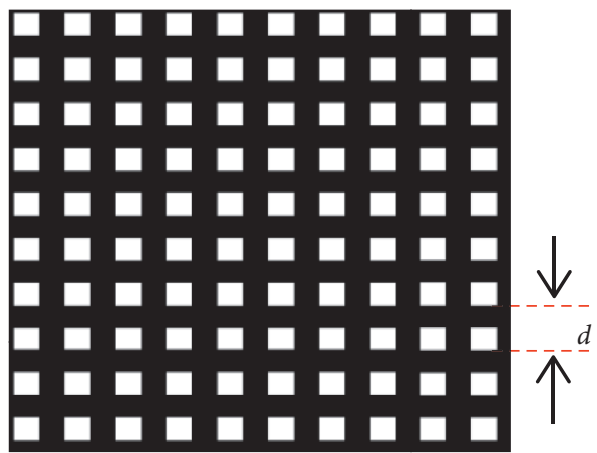

FIgURE 2: Schematic diagram of the two-dimensional square hole grating.

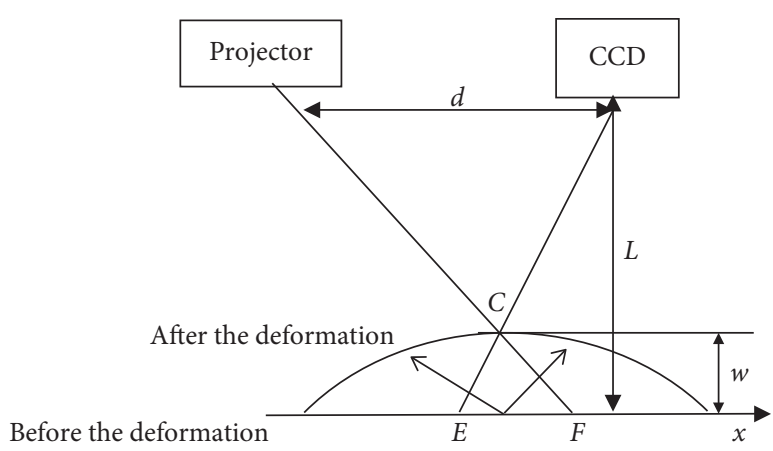

Figure 3: Geometry employed to determine the out-of-plane displacement.

where $k=M L / d$. For the given Talbot fringe projection system, $k$ is a constant and can be obtained by experimental calibration.

A digital image correlation (DIC) algorithm was used to obtain displacement maps at the surface of a deformed object by tracking the displacement of groups of pixels from a sequence of images acquired during deformation. DIC is a commonly used technique for deformation measurements that statistically correlate subsets of two fringes that capture the status of the surface of an object before and after deformation [31]. The basic principle of DIC is that before and after the deformation of the 
object, the movement of geometric points on its surface produces in-plane displacement. The displacement field can be calculated using the DIC algorithm which depends on the selected criterion [32]. Based on the combination of fringe projection and DIC techniques, out-of-plane and in-plane displacements can be simultaneously measured [33, 34]. In Reference [33], Felipe-Sesé et al. proposed a color encoding methodology based on the combination of fringe projection and 2D DIC techniques using a single-color CCD camera. In this case, the in-plane and out-of-plane displacements are simultaneously measured.

The cross-correlation function between the digital images of the surface of an object before and after deformation can be expressed as follows [35]:

$$
C(u, v)=\frac{\sum_{i}^{m} \sum_{j}^{m}\left[f\left(x_{i}, y_{j}\right)-\bar{f}\right]\left[g\left(x_{i}+u, y_{j}+v\right)-\bar{g}\right]}{\sqrt{\sum_{i}^{m} \sum_{j}^{m}\left[f\left(x_{i}, y_{j}\right)-\bar{f}\right]^{2}} \sqrt{\sum_{i}^{m} \sum_{j}^{m}\left[g\left(x_{i}+u, y_{j}+v\right)-\bar{g}\right]^{2}}},
$$

where $C$ is the maximum correlation factor, $f$ is the intensity image before the deformation, $g$ is the intensity image after deformation, and $u$ and $v$ are the integer-pixel displacements in the $x$ and $y$ directions, respectively, at any point on the image. The parameters $\bar{f}$ and $\bar{g}$ represent the average gray value of the image subregions. Equation (5) indicates that a subarea with an area of $m \times m$ pixels is identified on each of the images before and after the deformation to calculate the correlation coefficient, and $u$ and $v$ (which can assume the maximum value) are the displacements of the center point of the subarea. A gradient-based DIC algorithm can improve the accuracy of the approach. The correlation coefficient is expressed as follows [36]:

$$
C(u, v)=\frac{\left\{\sum_{i}^{m} \sum_{j}^{m}\left[f\left(x_{i}, y_{j}\right)-\bar{f}\right]\left[g\left(x_{i}+u+\Delta u, y_{j}+v+\Delta v\right)-\bar{g}\right]\right\}^{2}}{\left.\sum_{i}^{m} \sum_{j}^{m}\left[f\left(x_{i}, y_{j}\right)-\bar{f}\right)\right]^{2} \sum_{i}^{m} \sum_{j}^{m}\left[g\left(x_{i}+u+\Delta u, y_{j}+v+\Delta v\right)-\bar{g}\right]^{2}},
$$

where $\Delta u$ and $\Delta v$ represent the subpixel displacements. Take the Taylor expansion of $g\left(x_{i}+u+\Delta u, y_{j}+v+\Delta v\right)-\bar{g}$ and the first-order approximation. In consideration of $(\partial C / \partial \Delta u)=0,(\partial C / \partial \Delta v)=0$, we can obtain the following formula:

$$
\left[\begin{array}{l}
\Delta u \\
\Delta v
\end{array}\right]=\left[\begin{array}{ll}
B & C \\
E & H
\end{array}\right]^{-1}\left[\begin{array}{l}
A \\
D
\end{array}\right]
$$

where

$$
\begin{aligned}
A & =\sum_{i=1}^{m} \sum_{j=1}^{m} F G \sum_{i=1}^{m} \sum_{j=1}^{m} G G_{x}-\sum_{i=1}^{m} \sum_{j=1}^{m} F G_{x} \sum_{i=1}^{m} \sum_{j=1}^{m} G^{2}, \\
B & =\sum_{i=1}^{m} \sum_{j=1}^{m} F G_{x} \sum_{i=1}^{m} \sum_{j=1}^{m} G G_{x}-\sum_{i=1}^{m} \sum_{j=1}^{m} G_{x}^{2} \sum_{i=1}^{m} \sum_{j=1}^{m} F G, \\
C & =2 \sum_{i=1}^{m} \sum_{j=1}^{m} F G_{x} \sum_{i=1}^{m} \sum_{j=1}^{m} G G_{y}-\sum_{i=1}^{m} \sum_{j=1}^{m} G G_{x} \sum_{i=1}^{m} \sum_{j=1}^{m} F G_{y}-\sum_{i=1}^{m} \sum_{j=1}^{m} G_{x} G_{y} \sum_{i=1}^{m} \sum_{j=1}^{m} F G \\
D & =\sum_{i=1}^{m} \sum_{j=1}^{m} F G \sum_{i=1}^{m} \sum_{j=1}^{m} G G_{y}-\sum_{i=1}^{m} \sum_{j=1}^{m} F G_{y} \sum_{i=1}^{m} \sum_{j=1}^{m} G^{2}, \\
E & =2 \sum_{i=1}^{m} \sum_{j=1}^{m} F G_{y} \sum_{i=1}^{m} \sum_{j=1}^{m} G G_{x}-\sum_{i=1}^{m} \sum_{j=1}^{m} G G_{y} \sum_{i=1}^{m} \sum_{j=1}^{m} F G_{x}-\sum_{i=1}^{m} \sum_{j=1}^{m} G_{x} G_{y} \sum_{i=1}^{m} \sum_{j=1}^{m} F G, \\
H & =\sum_{i=1}^{m} \sum_{j=1}^{m} F G_{y} \sum_{i=1}^{m} \sum_{j=1}^{m} G G_{y}-\sum_{i=1}^{m} \sum_{j=1}^{m} G_{y}^{2} \sum_{i=1}^{m} \sum_{j=1}^{m} F G \\
G\left(x_{i}, y_{j}\right) & =g\left(x_{i}+u, y_{j}+v\right)-\bar{g}, \\
G_{x} & =g_{x}-\bar{g}_{x}, \\
G_{y} & =g_{y}-\bar{g}_{y}, \\
F\left(x_{i}, y_{j}\right) & =f\left(x_{i}, y_{j}\right)-\bar{f},
\end{aligned}
$$




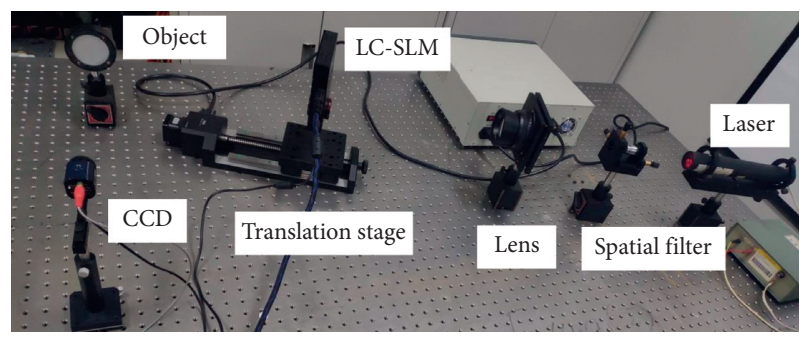

FIgURE 4: Experimental setup.

where $x$ and $y$ subscripts of variables are the partial derivatives.

\section{Experimental Results}

The experimental setup used to measure the in-plane and out-of-plane displacements is shown in Figure 4. A spatial filter and collimator are placed in the optical path of a He-Ne laser operated at $632.8 \mathrm{~nm}$. After passing through a circular aperture, the beam is to illuminate a transmissive LC-SLM. In our scheme, the LC-SLM (RSLM1024 from UPOLabs, with a resolution of 1024 pixels $\times 768$ pixels and a pixel size of $18 \mu \mathrm{m}$ ) is controlled using a personal computer (PC1), which is used to input the designed square Talbot array hologram $(a=20 \mu \mathrm{m}, d=120 \mu \mathrm{m})$ into the LC-SLM. The LCSLM is fixed on a precision translation stage. By moving the translation stage back and forth in the direction of the incident beam, a clear speckle pattern can be observed on the surface of the object. A personal computer (PC2) was connected to a CCD camera (MV-VDM130SC from Microvision with a resolution of 1280 pixels $\times 960$ pixels and a pixel size of $3.75 \mu \mathrm{m} \times 3.75 \mu \mathrm{m}$ ) to capture the fringe patterns on the sample's surface. The signal-to-noise ratio of the camera was greater than $52 \mathrm{~dB}$.

In the experiment, the test sample was a circular aluminum plate (10 $\mathrm{cm}$ in diameter and $3 \mathrm{~mm}$ in thickness). The plate was fixed at the periphery and centrally loaded, as shown in Figure 5. Out-of-plane deformation was achieved by fine tuning the micrometer screw rod (with a resolution of $1 \mu \mathrm{m})$ at the center of the back of the plate.

Figure 6 shows the Talbot fringe patterns on the surface of the test piece captured by the CCD camera before and after the object is loaded. In the figure, Figure 6(a) is the pattern before the object is deformed, and Figure 6(b) is the pattern after deformation of the object. The distance between the object and the LC-SLM is 6 times the Talbot distance $(27.31 \mathrm{~cm})$. Using the method proposed by Pan [29], the parameter $k$ is determined to be $1.77 \mu \mathrm{m} /$ pixel.

Figure 7 shows the in-plane and out-of-plane displacements obtained using the filtering technique and the DIC algorithm. This process is performed using MATLAB software. In the actual calculation using the DIC method, global searching is employed in the subregion match. The size of the subimage used in DIC to calculate the correlation coefficient at one point is $41 \times 41$ pixels, but the searching area has a size of $101 \times 101$ pixels.

The maximum out-of-plane displacement at the center of the sample that was obtained using DIC was $8.10 \mu \mathrm{m}$. Before

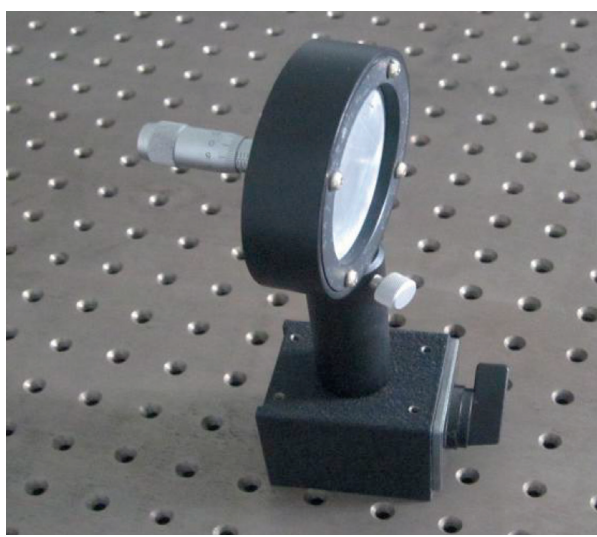

FIGURE 5: Schematic of the centrally loaded sample.

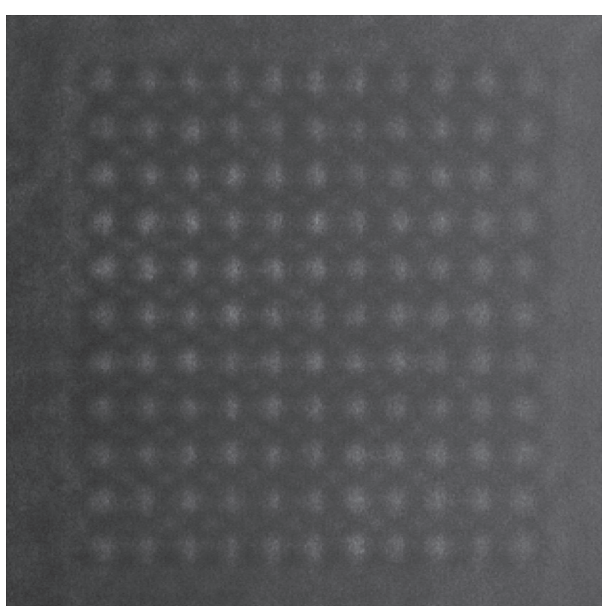

(a)

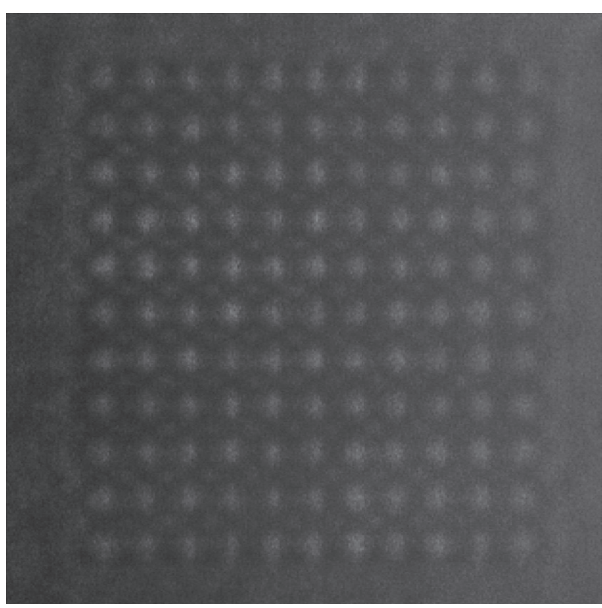

(b)

FIgURE 6: Experimental results. (a) Before the deformation. (b) After the deformation.

and after the deformation of the sample, the indication of the screw was read, and the maximum out-of-plane displacement of the sample was $8.50 \mu \mathrm{m}$. The maximum out-of-plane displacement measured using the screw was taken as the reference value (which can be regarded as the true value) to calculate the error, which was determined to be $4.70 \%$. 


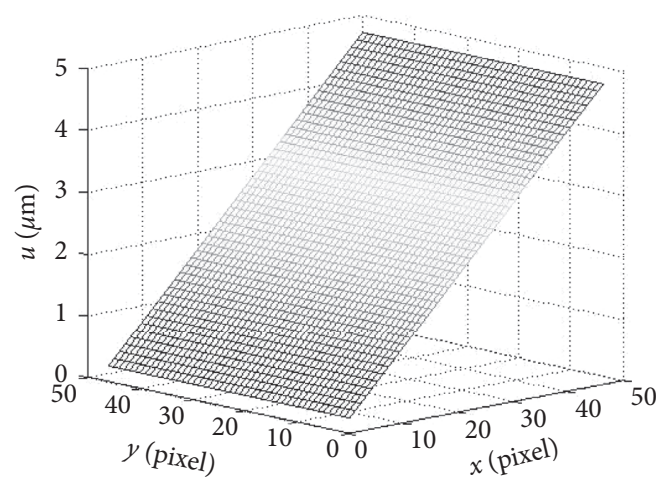

(a)

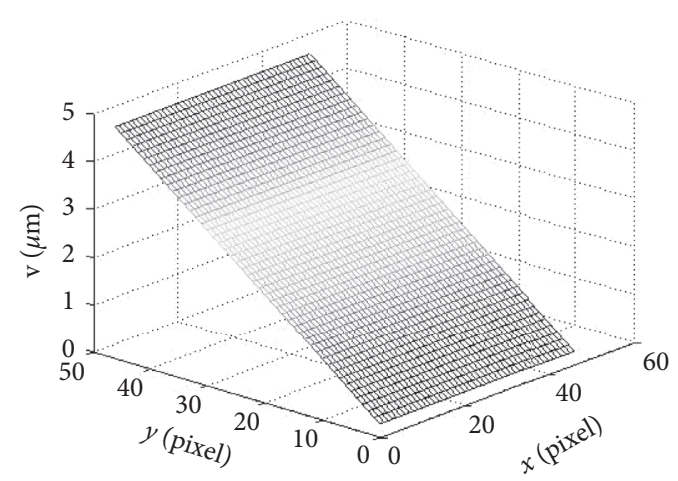

(b)

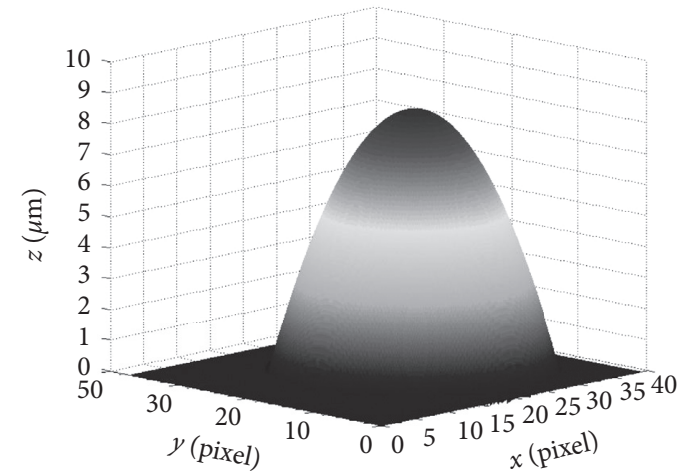

(c)

Figure 7: 3D displacement obtained using DIC. (a) $u$ component of the in-plane displacement. (b) $v$ component of the in-plane displacement. (c) Out-of-plane displacement component.

The experimental results show that the out-of-plane displacement of the sample can be obtained using Talbot fringe projection. This conclusion is consistent with the results presented in the literature [32], in which DIC is used to analyze out-of-plane deformation in turbid fluids, and the optical flow method is used to measure the out-of-plane component of the deformation phase [37].

\section{Conclusions}

In conclusion, we proposed a method for $3 \mathrm{D}$ displacement measurement using Talbot fringe projection. In this approach, a hologram of a square Talbot array was inputted to an LC-SLM screen and projected onto the sample. Images of the patterns on the surface of the sample before and after the deformation were captured using a CCD camera. In-plane and out-of-plane displacements were obtained using DIC. Instead of using a projection system to project a grating on the surface of the object for either shadow moiré or projection moiré analysis, projection can be performed without a lens using the Talbot effect.

\section{Data Availability}

The detailed experimental data used to support the findings of this study are available from the corresponding author upon request.

\section{Conflicts of Interest}

The authors declare that there are no conflicts of interest regarding the publication of this paper.

\section{Acknowledgments}

This work was supported by the Shandong Provincial Natural Science Foundation, China (nos. ZR2017LA010 and ZR201702090137), the National Science Foundation of China (61975099 and 11902317), and Tai' an City Science and Technology Development Plan (Guidance Plan) Project (2018GX0076).

\section{References}

[1] S. Zhang, "Absolute phase retrieval methods for digital fringe projection profilometry: a review," Optics and Lasers in Engineering, vol. 107, pp. 28-37, 2018.

[2] Z. Wang, Z. Zhang, N. Gao, Y. Xiao, F. Gao, and X. Jiang, "Single-shot 3D shape measurement of discontinuous objects based on a coaxial fringe projection system," Applied Optics, vol. 58, no. 5, p. A169, 2019.

[3] S. Xing and H. Guo, "Directly recognizing and removing the projector nonlinearity errors from a phase map in phaseshifting fringe projection profilometry," Optics Communications, vol. 435, pp. 212-220, 2019.

[4] L. Felipe-Sesé, E. López-Alba, P. Siegmann, and F. A. Díaz, "Integration of fringe projection and two-dimensional digital 
image correlation for three-dimensional displacements measurements," Optical Engineering, vol. 55, no. 12, Article ID 121711, 2016.

[5] R. Beermann, L. Quentin, E. Reithmeier, and M. Kästner, "Fringe projection system for high-temperature workpiecesdesign, calibration, and measurement," Applied Optics, vol. 57 , no. 15 , p. $4075,2018$.

[6] W. L. Gubbels and G. S. Schajer, "Development of 3-D digital image correlation using a single color-camera and diffractive speckle projection," Experimental Mechanics, vol. 56, no. 8, pp. 1327-1337, 2016.

[7] J. Pineda, A. G. Marrugo, and L. A. Romero, "Developing a robust acquisition system for fringe projection profilometry," Journal of Physics: Conference Series, vol. 1247, Article ID 012053, 2019.

[8] G. Hu, X. Zhou, G. Zhang et al., "Multiple laser stripe scanning profilometry based on microelectromechanical systems scanning mirror projection," Micromachines, vol. 10, no. 1, p. 57, 2019.

[9] M. Ratnam, M. Saxena, and S. S. Gorthi, "Circular fringe projection technique for out-of-plane deformation measurements," Optics and Lasers in Engineering, vol. 121, pp. 369-376, 2019.

[10] X. Liu and J. Kofman, "Real-time 3D surface-shape measurement using background-modulated modified Fourier transform profilometry with geometry-constraint," Optics and Lasers in Engineering, vol. 115, pp. 217-224, 2019.

[11] Y. Ding, K. Peng, M. Yu et al., "Fringe order correction for the absolute phase recovered by two selected spatial frequency fringe projections in fringe projection profilometry," Review of Scientific Instruments, vol. 88, no. 8, Article ID 083104, 2017.

[12] Y. Wan, Y. Cao, C. Chen et al., "Active phase error suppression for color phase-shifting fringe projection based on hue pre-correction," Optics \& Laser Technology, vol. 118, pp. 102-108, 2019.

[13] C. Zuo, S. Feng, L. Huang, T. Tao, W. Yin, and Q. Chen, "Phase shifting algorithms for fringe projection profilometry: a review," Optics and Lasers in Engineering, vol. 109, pp. 23-59, 2018.

[14] J. Wang and Y. Yang, "A wavelet ridge extraction method employing a novel cost function in two-dimensional wavelet transform profilometry," AIP Advances, vol. 8, no. 5, Article ID 055020, 2018.

[15] R. Zhao, Y. Huang, J. Wang et al., "Image-spherizing-based planeness detecting method for a micro-channel plate," $A p$ plied Optics, vol. 58, no. 3, pp. 554-560, 2019.

[16] G. S. Spagnolo, D. Ambrosini, and D. Paoletti, "Displacement measurement using the Talbot effect with a Ronchi grating," Journal of Optics A: Pure and Applied Optics, vol. 4, no. 6, pp. S376-S380, 2002.

[17] P. Chavel and T. C. Strand, "Range measurement using Talbot diffraction imaging of gratings," Applied Optics, vol. 23, no. 6, pp. 862-871, 1984.

[18] G. S. Spagnolo and D. Ambrosini, "Talbot effect application: measurement of distance with a Fourier-transform method," Measurement Science and Technology, vol. 11, no. 1, pp. 77-82, 2000.

[19] P. Rodriguez-Montero, D. Sánchez-de-la-Llave, and S. Mansurova, "Electro-optical processor for measuring displacement employing the Talbot and the nonsteady-state photo-electromotive force effects," Optics Letters, vol. 39, no. 1, pp. 104-107, 2014.

[20] X. Xu, S. Zhou, and G. Huang, "Measurement of micro-angle based on the Talbot effect of 2D grating," in Proceedings of
SPIE, vol. 10818, Article ID 1081813, Beijing, China, November 2018.

[21] H. O. Carmesin and D. Goldbeck, "Depth map by convergent 3D-Talbot-interferometry," Optik, vol. 108, no. 3, pp. 101-116, 1998.

[22] S. Mirza and C. Shakher, "Surface profiling using phase shifting Talbot interferometric technique," Optical Engineering, vol. 44, no. 1, Article ID 013601, 2005.

[23] J. R. Leger and M. A. Snyder, "Real-time depth measurement and display using Fresnel diffraction and white-light processing," Applied Optics, vol. 23, no. 10, pp. 1655-1670, 1984.

[24] K. Patorski, "The self-imaging phenomenon and its applications," in Progress in Optics, E. Wolf, Ed., pp. 1-108, Elsevier, Amsterdam, Netherlands, 1989.

[25] M. Dashtdar, A. Mohammadzade, and S. M.-A. HosseiniSaber, "Measurement of roughness based on the Talbot effect in reflection from rough surfaces," Applied Optics, vol. 54, no. 16, pp. 5210-5215, 2015.

[26] M. Dashtdar and S. M. A. H. Saber, "Determination of the rough interface parameters using the self-imaging effect," Journal of the Optical Society of America A, vol. 30, no. 11, pp. 2416-2421, 2013.

[27] S. Agarwal and C. Shakher, "In-plane displacement measurement by using circular grating Talbot interferometer," Optics and Lasers in Engineering, vol. 75, pp. 63-71, 2015.

[28] R. Rodriguez-Vera, D. Kerr, and F. Mendoza-Santoyo, "3-D contouring of diffuse objects by talbot-projected fringes," Journal of Modern Optics, vol. 38, no. 10, pp. 1935-1945, 1991.

[29] R. Rodriguez-Vera, J. A. Rayas, F. Mendoza-Santoyo et al., "Advances on Talbot projection profilometry," in Proceedings of the AIP Conference, vol. 1236, pp. 409-418, Portland, OK, USA, July 2010.

[30] B. Pan, H. Xie, J. Gao, and A. Asundi, "Improved speckle projection profilometry for out-of-plane shape measurement," Applied Optics, vol. 47, no. 29, pp. 5527-5533, 2008.

[31] G. M. Hassan, "Digital image correlation for discontinuous displacement measurement using subset segmentation," Optics and Lasers in Engineering, vol. 115, pp. 208-216, 2019.

[32] A. Aranda, N. Amigo, C. Ihle et al., "Digital image correlation applied to the calculation of the out-of-plane deformation induced by the formation of roll waves in a non-Newtonian fluid," Optical Engineering, vol. 55, no. 6, Article ID 064101, 2016.

[33] L. Felipe-Sesé, P. Siegmann, F. A. Díaz, and E. A. Patterson, "Simultaneous in-and-out-of-plane displacement measurements using fringe projection and digital image correlation," Optics and Lasers in Engineering, vol. 52, pp. 66-74, 2014.

[34] H. Yan and B. Pan, "Three-dimensional displacement measurement based on the combination of digital holography and digital image correlation," Optics Letters, vol. 39, no. 17, pp. 5166-5169, 2014.

[35] P. Sun, K. Shi, M. Sun et al., "Evaluation of 3D displacement components by combining DSCM with ESPI," in Proceedings of SPIE, vol. 10023, pp. 100231-100236, Beijing, China, November 2016.

[36] C. Guang, Q. Feng, K. Ding et al., "Subpixel displacement measurement method based on the combination of particle swarm optimization and gradient algorithm," Optical Engineering, vol. 56, no. 10, Article ID 104101, 2017.

[37] R. Zhao and P. Sun, "Deformation-phase measurement by optical flow method," Optics Communications, vol. 371, pp. 144-149, 2016. 